\title{
AGO Recommendations for the Diagnosis and Treatment of Patients with Advanced and Metastatic Breast Cancer: Update 2015
}

\author{
Cornelia Liedtke ${ }^{\mathrm{a}}$ Marc Thill ${ }^{\mathrm{b}}$ Volker Hanf ${ }^{\mathrm{c}}$ \\ Florian Schütz ${ }^{\mathrm{d}}$ on behalf of the AGO Breast Committee* \\ ${ }^{a}$ Klinik für Frauenheilkunde und Geburtshilfe, Universitätsklinikum Schleswig-Holstein/Campus Lübeck, Germany; \\ ${ }^{b}$ Klinik für Gynäkologie und Geburtshilfe, Agaplesion Markus Krankenhaus, Frankfurt/M., Germany; \\ cFrauenklinik, Klinikum Fürth, Germany; \\ dUniversitätsfrauenklinik Heidelberg, Germany
}

\section{Keywords}

Guidelines - Locoregional relapse .

Metastatic breast cancer - Targeted therapy

\section{Introduction}

For the last 14 years, the Breast Committee of the Arbeitsgemeinschaft Gynäkologische Onkologie (German Gynecological Oncology Group, AGO) has issued annually updated evidencebased recommendations for the diagnosis and treatment of patients with early and metastatic breast cancer. The AGO Breast Committee consists of 45 gynecological oncologists specialized in breast cancer and interdisciplinary members specialized in pathology, radiological diagnostics, medical oncology, and radiotherapy. Each update is performed/assembled in accordance with documented rules by thoroughly reviewing and scoring the recent publications chapter by chapter for i) their scientific validity (Oxford

${ }^{*}$ Members of the 'AGO Breast Committee' in alphabetical order: Ute-Susann Albert, Frankfurt/M.; Ingo Bauerfeind, Landshut; Joachim Bischoff, Dessau; Jens Uwe Blohmer, Berlin; Klaus Brunnert, Osnabrück; Peter Dall, Lüneburg; Ingo J. Diel, Mannheim; Prof. Dr. Tanja Fehm, Düsseldorf; Nikos Fersis, Bayreuth; Michael Friedrich, Krefeld; Kay Friedrichs, Hamburg; Bernd Gerber, Rostock; Volker Hanf, Fürth; Nadia Harbeck, Munich; Jens Huober, Ulm; Christian Jackisch, Offenbach; Wolfgang Janni, Ulm; Hans H. Kreipe, Hanover; Sherko Kümmel, Essen; Thorsten Kühn, Esslingen; Cornelia Liedtke, Lübeck; Sibylle Loibl, Neu-Isenburg; Michael Lux, Erlangen; Hans-Joachim Lück, Hanover; Nicolai Maas, Lübeck; Volker Möbus, Frankfurt/M.; Volkmar Müller, Hamburg; Gunter von Minckwitz, Neu-Isenburg; Christoph Mundhenke, Kiel; Ulrike Nitz, Mönchengladbach; Mahdi Rezai, Düsseldorf; Achim Rody, Lübeck; Anton Scharl, Amberg; Rita Schmutzler, Cologne; Marcus Schmidt, Mainz; Andreas Schneeweiss, Heidelberg; Florian Schütz, Heidelberg; Ingrid Schreer, Kiel; H. Peter Sinn, Heidelberg; Erich Solomayer, Homburg; Rainer Souchon, Berlin; Elmar Stickeler, Freiburg i.Br.; Marc Thill, Frankfurt/M.; Christoph Thomssen, Halle/Saale; Michael Untch, Berlin.

The members of the AGO Breast Committee are also listed in the appendix accessible at www.karger.com $/$ ?DOI=431248

\section{KARGER}

Fax +497614520714

\section{(๑) 2015 S. Karger GmbH, Freiburg}

$1661-3791 / 15 / 0103-0199 \$ 39.50 / 0$
Level of Evidence, LoE; www.cebm.net [1]) and ii) their clinical relevance (AGO grades of recommendation) (table 1). All AGO Breast Committee members have declared their potential conflicts of interest. Here, we present the 2015 update of these guidelines, focusing on relevant changes introduced this year. The full version of the updated 2015 slide set is available online as a PDF file [2] in English and German language. Moreover, a version for patients is also available at www.ago-online.de.

\section{Bone Health and Osteo-Oncology}

\section{Treatment of Bone Metastases}

Recommendations regarding the prevention or treatment of bone metastases have remained largely unchanged compared to last year's recommendations. One issue still being debated is the optimal schedule for patients with bone metastases undergoing longterm antiresorptive treatment. In fact, recent results suggest that treatment intervals may be extended after 1 year of bisphosphonate therapy. For instance, the recently presented OPTIMIZE-2 phase III trial demonstrates that dosing can be safely scaled back from every 4 weeks to every 12 weeks [3]. In this trial, 403 patients who had received at least 9 doses of bisphosphonates were randomly assigned to receive zoledronic acid (4 mg intravenously) every 4 weeks versus every 12 weeks for an additional year. The skeletal event rates were $22 \%$ among women receiving zoledronic acid every 4 weeks versus $23 \%$ for those with a 12 -week schedule, indicating that the effectiveness of the bisphosphonate is not compromised by a 3-monthly schedule. Therefore, extension of treatment intervals may be an option in some cases ( $\mathrm{LoE} 1 \mathrm{ba} / \mathrm{B} / \mathrm{AGO}+$ ).

Although there is a significant number of patients experiencing recurrent or persistent metastatic bone pain after bone irradiation, 
Table 1. AGO grades of recommendation
This investigation or therapeutic intervention is highly beneficial for patients, can be recommended without restriction, and should be performed.

$+\quad$ This investigation or therapeutic intervention is of limited benefit for patients and can be performed.

+/- This investigation or therapeutic intervention has not shown benefit for patients and may be performed only in individual cases. According to current knowledge, a general recommendation cannot be given.

This investigation or therapeutic intervention can be of disadvantage for patients and might not be performed.

-/ $\quad$ This investigation or therapeutic intervention is of clear disadvantage for patients and should be avoided or omitted in any case. treatment options are limited. Hurwitz et al. [4] recently presented the results of a randomized phase III trial investigating the safety and efficacy of magnetic resonance-guided focused ultrasound surgery (MRgFUS) for this indication. The response rate for the primary endpoint (self-reported pain score without increase in pain medication 3 months after treatment) was $64.3 \%$ in the MRgFUS arm and $20.0 \%$ in the placebo arm $(\mathrm{p}<0.001)$. Therefore, MRgFUS may be an option among patients with metastatic bone pain after failure of standard treatment ( $\mathrm{LoE} 1 \mathrm{~b} / \mathrm{B} / \mathrm{AGO}+)$.

\section{Treatment of Therapy-Induced Osteoporosis}

During the previous 12 months, no relevant novel findings with respect to medical treatment of bone loss could be observed, despite an increasing body of evidence regarding this issue. It has to be acknowledged though that atypical fractures particularly of the femur shaft must be regarded as noteworthy sequelae of long-lasting treatment with bisphosphonates. While the hazard ratio for these atypical fractures is very high in comparison to non-exposed people, and particularly so in women, the absolute risk of 11 (confidence interval (CI): 7-14) fractures per 10,000 person-years of use is still low. The risk decreases by $70 \%$ for each year since last use. Risk estimates may be affected by the type of bisphosphonate used, and the risk has been observed to decrease rapidly after cessation [5]. Based on the available data, one may conclude that bisphosphonates are effective in the primary prevention of symptomatic fragility fractures during the first few years of use in female patients with osteoporosis who are less than 80 years of age. Prolongation of bisphosphonate treatment, however, does not appear to improve the initial benefit any further, but the risk of atypical femoral fracture increases with every year of use. Bisphosphonate treatment should therefore be limited with regard to both treatment duration and indication for treatment.

\section{Follow-Up of Breast Cancer}

One of the major goals of breast cancer follow-up is the early detection of curable breast cancer events, i.e. breast or locoregional recurrence. The early detection of symptomatic metastases is desirable; however, in terms of the early detection of asymptomatic metastases, data are inconsistent and most importantly do not suggest a survival benefit. Beyond improvement of survival, additional issues like improvement of quality of life, physical performance, and reduction of treatment-related side effects are important (LoE 2b/B/ AGO +). In addition, re-evaluation of current adjuvant therapies and assessment or improvement of treatment compliance (especially endocrine therapy) should be part of follow-up ( $\mathrm{LoE} 5 / \mathrm{D} / \mathrm{AGO}++$ ). With regard to this, it should be pointed out that every patient has the right to obtain a second opinion ( $\operatorname{LoE} 2 \mathrm{c} / \mathrm{B} / \mathrm{AGO}++$ ) as well as to undergo genetic counseling, hormone replacement, prophylactic surgery, and breast reconstruction ( $\operatorname{LoE} 2 \mathrm{c} / \mathrm{C} / \mathrm{AGO}+$ ). Further issues such as pregnancy, contraception, sexuality, quality of life, menopausal symptoms, and specific psychological aspects should be addressed proactively ( $\mathrm{LoE} 4 / \mathrm{C} / \mathrm{AGO}+$ ).

Life style modifications (cessation of smoking, diet, reduced alcohol consumption, and physical activity) and interventions with regard to co-morbidities (diabetes) are further important aspects of follow-up. From a patient's perspective, examination of the breast, reassurance, guidance with answering questions, evaluation of treatment with side effects, and psychosocial support are relevant.

Most importantly, follow-up examinations of asymptomatic patients in routine situations should not contain tumor marker measurements, liver ultrasound, bone scans, X-ray, computed tomography or positron emission tomography scans, and monitoring of circulating tumor cells (CTC). Imaging to detect curable events (mammography, ultrasound, and in specific situations magnetic resonance imaging) in combination with self-examination and physical examination is recommended. In this context, screening for second malignancies according to guidelines (e.g. colorectal, endometrial, ovarian, cervical cancer, and lymphoma) is meaningful.

\section{Locoregional Recurrence}

In patients with locoregional relapse, pretherapeutic biopsy to re-assess histology as well as estrogen receptor (ER), progesterone receptor (PR), and human epidermal growth-factor receptor 2 (HER2) status is strongly recommended (LoE 3b/B/AGO ++) [6]. Besides known clinicopathological factors, obesity is also associated with poor prognosis and breast cancer-specific survival, regardless of menopausal status and starting time of obesity. This was shown in a recent meta-analysis of 82 studies $(n=213,075)$ (LoE 1b) [7]. In addition, high-risk patients according to multigene assays are also at increased risk for locoregional relapse $[8,9]$. Clinical consequences, however, of these initial reports remain to be determined.

With regard to surgical options, sentinel lymph node dissection (SLND) after prior sentinel node excision may be feasible in case of cN0 status ( $\operatorname{LoE} 1 \mathrm{~b} / \mathrm{B} / \mathrm{AGO}+/$-). Axillary dissection should not be performed in cases with cN0 disease and lack of sentinel lymph node detection. In a recent Danish case series, SLND was possible in 72/144 patients (50\%). The detection rate after prior SLND (66\%) was significantly higher than after prior axillary dissection (34\%) [10]. 
With regard to systemic therapy, the CALOR trial ( $\mathrm{n}=163$; median follow-up 4.9 years) demonstrated significant benefit of postoperative chemotherapy in patients with fully excised locoregional relapse, particularly in the case of ER-negative disease (LoE 2b/B/ AGO +). 5-year disease-free survival rates were 69\% (95\% CI 5679 ) and 57\% (95\% CI 44-67) with and without chemotherapy, respectively (hazard ratio $0.59 ; 0.35-0.99 ; \mathrm{p}=0.046$ ) [11]. If chemotherapy is planned, a preoperative approach may be considered. In cases with HER2-positive disease, chemotherapy in combination with HER2-targeted therapy is an option (LoE 5/D/AGO +). It needs to be emphasized that patients with inoperable locoregional relapse were included in the pertuzumab registration trial CLEOPATRA, and thus the study results may also be relevant for this patient cohort. In patients with ER-positive locoregional relapse following complete resection (R0), endocrine therapy is considered standard (LoE 2b/B/AGO ++).

\section{Endocrine and Targeted Therapy in Metastatic Breast Cancer}

Endocrine therapy is the backbone of and first choice for the treatment of hormone receptor (HR)-positive metastatic breast cancer. In order to reliably determine the receptor status upon diagnosis of metastatic breast cancer, it is encouraged to biopsy and immunohistochemically stain the metastatic site whenever possible. Pooled relative discordance rates between primary tumors and metastatic disease for ER, PR, and HER2 status have been reported in $20 \%$ (95\% CI $16-35 \%), 33 \%$ (95\% CI $29-38 \%)$, and $8 \%$ (95\% CI 6-10\%) of cases, respectively [12]. However, even if analyses showed persisting HR positivity, tumor cells may have generated resistance against endocrine treatment.

In peri- and premenopausal patients, the induction of ovarian function suppression (gonadotropin-releasing hormone $(\mathrm{GnRH})$ analogue or luteinizing hormone analogue) is the first step in endocrine treatment. Whenever possible, it should be combined with tamoxifen $20 \mathrm{mg} /$ day [13]. GnRH analogue can be combined with an aromatase inhibitor (AI) if there are contraindications for tamoxifen. However, monotherapy with ovarian function suppression or tamoxifen alone also represents an option but is commonly not considered standard of care.

In postmenopausal patients, endocrine treatment should be selected depending on previous medication and relapse-free interval. The first choice of endocrine treatment should be an AI if either tamoxifen has already been used in the primary setting or an AI treatment was terminated more than 1 year ago [14]. If the cancer progressed on therapy with nonsteroidal AI (letrozole or anastrozole) or if an AI therapy was terminated less than 1 year ago, 2 distinct options for endocrine treatment may be considered: i) high dose fulvestrant (500 mg every 4 weeks) [15] or ii) a combination of exemestane plus everolimus [16] (fig. 1). In the case of disease progression on either 1 of these 2 agents at a later stage, the other agent may be used. Importantly, in selected patients, tamoxifen may be given in cases of oligometastatic or asymptomatic disease before chemotherapy is considered.

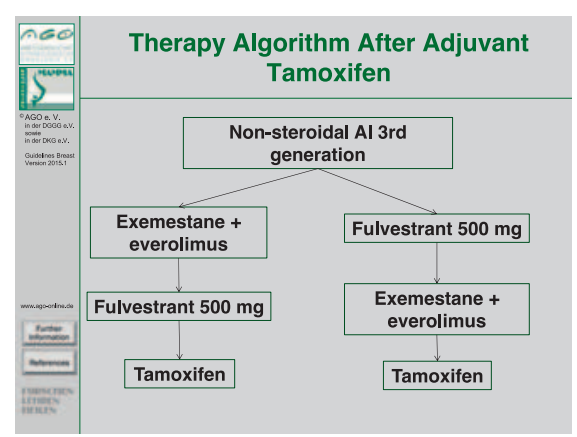

Fig. 1. Treatment recommendations for postmenopausal patients with metastatic breast cancer.

Given the results of a randomized phase II study, in which the addition of the cyclin-dependent kinase 4/6 inhibitor, palbociclib, to letrozole remarkably and significantly improved progression-free survival (PFS) in women with advanced ER-positive and HER2-negative breast cancer, use of this regimen may represent a novel endocrine treatment option in the future [17]. This combination despite license in the US is not yet licensed in Europe and therefore cannot be recommended for use in clinical routine ( $\mathrm{LoE} 2 \mathrm{~b} / \mathrm{B} / \mathrm{AGO}+/-$ ). Furthermore, given that the data cited above is limited to the phaseII setting, additional provide positive efficacy data before this agent shall enter daily clinical management outside of clinical trials.

In the LEA study, bevacizumab was added to first-line endocrine therapy with letrozole or fulvestrant. The study failed to demonstrate a significant increase in PFS in postmenopausal women with advanced HER2-negative and HR-positive breast cancer [18]. Therefore, initiation of bevacizumab in parallel to endocrine therapy in the case of disease progression is not recommended ( $\mathrm{LoE} 1 \mathrm{ba} / \mathrm{B} / \mathrm{AGO}-$-).

For patients who are treated with first-line chemotherapy in combination with bevacizumab and discontinue chemotherapy without evidence of progression, continuation of bevacizumab therapy in combination with second-line endocrine therapy is recommended until disease progression or toxicity ( $\operatorname{LoE} 2 \mathrm{ba} / \mathrm{B} / \mathrm{AGO}+$ ).

According to clinical symptoms and endocrine responsiveness, endocrine treatment should be stopped, and chemotherapy should be considered for further treatment. However, this should be discussed individually with each patient as part of shared decisionmaking. In HER2-positive/HR-positive advanced breast cancer, a combination of anastrozol or letrozole with trastuzumab or letrozole with lapatinib can be considered as a choice [19]. However, PFS was quite short in these clinical trials. Combination therapies of chemotherapy and HER2-directed treatment should be considered as a more effective option.

\section{Chemotherapy with or without Targeted Drugs in Metastatic Breast Cancer}

Treatment strategies in metastatic breast cancer are based on tumor biology with the choice of treatment depending on HR and HER2 status. Other parameters guiding treatment selection are i) possible combination with targeted agents; ii) previous treatments (and their toxicities); iii) aggressiveness of the disease and location of metastases; iv) biologic age; v) comorbidities (including organ 
Fig. 2. Duration of cytotoxic therapy in patients with metastatic breast cancer.

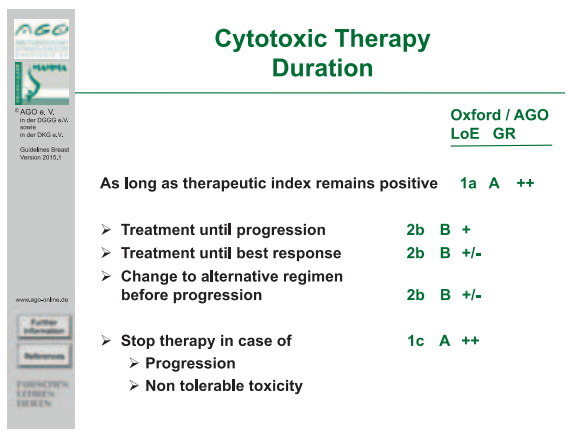

dysfunction); and vi) patient preference and expectations. In HRpositive tumors, endocrine therapy is generally preferred. If the leading site of metastases has been proven to be HR-negative (preferentially diagnosed by biopsy of at least 1 metastatic lesion) or if the course of the disease suggests endocrine resistance or urgent need for response, cytotoxic chemotherapy is indicated (LoE 1a/A/ $\mathrm{AGO}++$ ). This is in accordance with the recently published Second International Consensus Guidelines for Advanced Breast Cancer (ABC 2) [20]. Monotherapy is preferred to polychemotherapy in non-life-threatening situations, as combination therapy increases response rates but not overall survival (OS) [21]. It is recommended to treat as long as the therapeutic index remains positive ( $\mathrm{LoE} 1 \mathrm{a} / \mathrm{A} /$ $\mathrm{AGO}++$ ). Assuming a positive therapeutic index, the preferred option is to treat until progression ( $\mathrm{LoE} 2 \mathrm{~b} / \mathrm{B} / \mathrm{AGO}+)$, compared with stopping treatment at best response ( $\mathrm{LoE} 2 \mathrm{~b} / \mathrm{B} / \mathrm{AGO}+/-)$ (fig. 2).

A change of chemotherapy agents before tumor progression is recommended only as an exception (LoE $2 \mathrm{~b} / \mathrm{B} / \mathrm{AGO}+/-)$, although 1 trial showed a benefit of capecitabine plus bevacizumab therapy compared to bevacizumab alone after a taxane/bevacizumab therapy [22].

Monitoring of treatment response should be performed by assessment of the tumor burden at baseline and approximately every 2-3 months, i.e. every 2-4 cycles of treatment. In slow-growing disease, longer intervals are reasonable $(\mathrm{AGO}++)$.

The presence of CTC determined with the standardized Cell Search ${ }^{\circledR}$ system (Janssen Diagnostics South Raritan, NJ, USA) is an adverse prognostic factor for outcome, and CTC dynamics are an early predictor of therapy response [23]. Given the unclear consequences, CTC determination is recommended preferentially within clinical trials (LoE 1b/A/AGO +) [24].

In some settings, e.g. in triple-negative breast cancer (TNBC) or other aggressive progression, combination of chemotherapy with bevacizumab may be used as this has been shown to improve response rates and PFS, albeit not survival (LoE 2b/B/AGO +) [25]. Treatment with bevacizumab should usually be stopped in the case of disease progression, although 1 trial demonstrated a PFS benefit for the continuation with new lines of chemotherapy [26].

Platinum-based chemotherapy may be useful as treatment, especially in TNBC (LoE 1b/B/AGO +/-). Platinum compounds seem to be especially active in patients with BRCA mutations, and therefore a higher level of recommendation in this group is justified $(\mathrm{LoE} 2 \mathrm{~b} / \mathrm{B} / \mathrm{AGO}+)$ [27].

The most substantial progress on the past years has been achieved in HER2-overexpressing disease. Pertuzumab in combination with trastuzumab and docetaxel has shown benefit regarding both PFS and OS as first-line therapy compared to docetaxel and trastuzumab alone [28] and is recommended as first-line therapy (LoE 1b/A/ AGO ++). Furthermore, trastuzumab emtansine (T-DM1) is approved as a further HER2-targeted treatment option in metastatic breast cancer and has shown to enhance treatment efficacy (with respect to PFS and OS) in patients pretreated with trastuzumab either in comparison to lapatinib and capecitabine or in heavily pretreated patients compared with treatment of physician's choice [29, 30]. Therefore, T-DM1 represents a recommended treatment option for patients with HER2-positive metastatic breast cancer in i) the firstline setting diagnosed $<6$ months after adjuvant therapy using taxane and trastuzumab (LoE 1b/B/AGO ++) or ii) at second or further lines of therapy after trastuzumab treatment $(\mathrm{LoE} 1 \mathrm{~b} / \mathrm{A} / \mathrm{AGO}++)$.

\section{Specific Sites of Metastasis}

Specific sites of breast cancer metastasis are liver, lung, pleura, pericardium, peritoneum, bone marrow, and any soft tissue. Other rare locations like adrenals, ovaries, uterus, stomach, colon, or placenta have also been reported; however, in such rare cases, controlled trials are not feasible, and treatment options must be discussed on an individual basis.

Management of primary stage IV breast cancer should focus primarily on systemic therapy, given that the impact of the extent of local treatment on patient survival is still a matter of debate. Although some trials suggested an association between local treatment (surgery or radiotherapy) of the primary tumor and prolonged survival, recent reports do not observe these observations $[31,32]$. Therefore, controversy remains as to i) whether these results reflect a selection of women with good prognosis for primary site therapy; ii) what fraction of women in published studies were diagnosed with metastatic disease just after surgery; iii) whether specific subsets of metastases and biological subtypes would derive greater benefit; and iv) whether local therapy has been performed appropriately with regard to timing and extent. If surgery of the primary tumor is performed in the metastatic setting, local excision or mastectomy should be done with tumor-free margins [33, 34]. Axillary surgery is only indicated for bulky disease.

Systemic treatment of metastatic disease is the therapy of choice. Before treatment, metastases should be confirmed by histology to reevaluate diagnosis, HR status, and HER2 status. Discordance regarding these markers may occur in up to $45 \%$ of patients and may have an impact on systemic treatment. If surgery for distant metastases is considered, good overall health, oligometastasis, and a long time between primary treatment and the occurrence of metastases are all favorable factors regarding outcome. Resection of liver metastases may be performed after histological verification if R0 resection is feasible, no extrahepatic metastases were present, and tumor biology shows a HR-positive breast cancer responding well to former systemic therapy $[35,36]$. Other procedures like regional radiotherapy, thermoablation, or chemoembolization are also possible in individual cases $[37,38]$. 
For patients with pulmonary metastases, the LoE for a curative approach is low, but some patients might benefit from metastasectomy followed by appropriate systemic treatment [39]. In accordance with the treatment of liver metastases, resection of lung metastases should only be performed if R0 resection is feasible and if histological verification was done. The timing of any local intervention may be critical; resection before progression is associated with better outcome.

About $10 \%$ of all breast cancer patients develop malignant pleural effusion (MPE). In almost 50\% of MPE cases, it is the first sign of metastatic disease, resulting in dyspnea and reduced subjective well-being. It should be treated in symptomatic cases exclusively. Thoracoscopy with talcum pleurodesis (i.e. video-assisted thoracoscopy, VATS) is the treatment of choice for MPE. Other sclerosing but less often used agents are bleomycin, doxycycline, and mitoxantrone [40]. Continuous pleural drainage with indwelling pleural catheters is a well-tolerated and safe treatment alternative for patients who are not candidates for VATS. Catumaxomab is not yet recommended for MPE.

Overall, $3 \%$ of breast cancer patients will suffer from malignant ascites. Management of ascites takes place in the context of palliative care and aims at improving the quality of life of these patients. Patients with symptomatic ascites should undergo drainage. Local antibody therapy with catumaxomab remains an option in individual cases [41].

Malignant pericardial effusion and cardiac tamponade remain rare metastatic locations in patients with breast cancer. In symptomatic patients, drainage and pericardial fenestration are probably the treatment options of choice. For individual patients, VATS or ultrasound-guided puncture with instillation of mitoxantrone or bleomycin may be an alternative [42]

The choice between supportive care or specific anticancer treatment for poor performance status breast cancer patients with multimetastatic disease and pancytopenia due to bone marrow involvement often remains a clinical and human dilemma. Depending on the underlying cancer biology, endocrine therapy or chemotherapy or antibody treatment options should be reconsidered [43]. It has been reported that aggressive combination treatment regimens were effective since most patients showed improved marrow function after chemotherapy, and prolonged survival could be possible. Again, no significant changes to the recommendations in this chapter have been considered necessary.

\section{Central Nervous System Metastases in Breast Cancer}

Breast cancer represents the second most frequent origin of brain metastases (BM). It is estimated that $10-30 \%$ of patients with metastatic breast cancer are diagnosed with BMs. Due to i) better detection of subclinical disease with improved imaging techniques and ii) longer systemic control of extracerebral metastases particularly among patients with HER2-positive breast cancer, the incidence of breast cancer BMs is increasing. Therefore, the optimal management of BMs is of utmost importance (fig. 3). However, studies on BMs frequently involve various tumor entities; therefore, the data regarding the treatment of BMs specifically in breast cancer patients is lim-

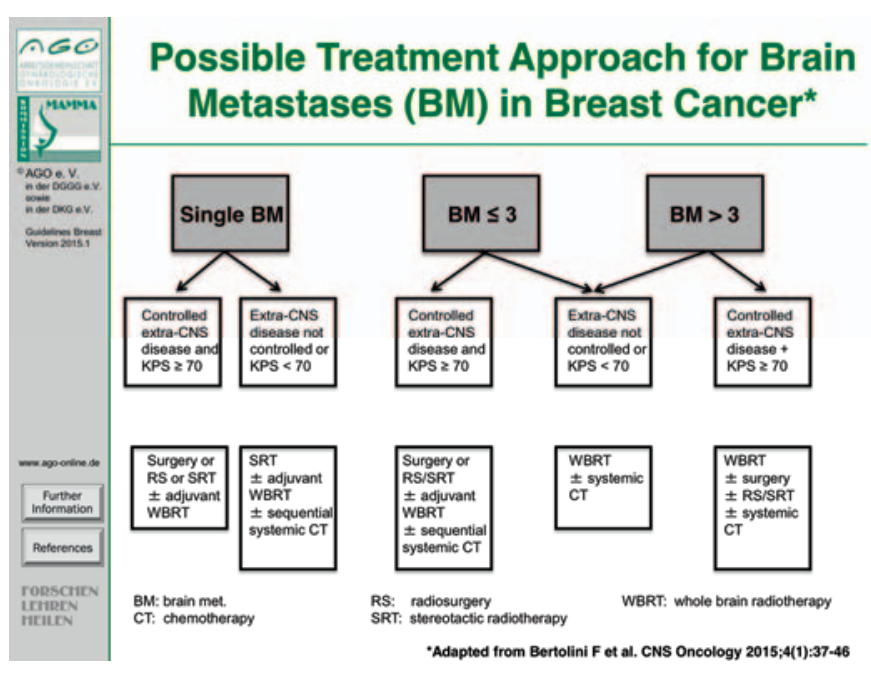

Fig. 3. Treatment options for breast cancer metastases and brain metastases.

ited. For optimization of treatment of breast cancer patients with BMs, a diagnosis-specific graded prognostic assessment was developed which takes into account the Karnofsky performance score, the biologic subtype, number of BMs, and age [44]. For patients with multiple BMs, the treatment of choice is still whole-brain radiotherapy (WBRT). Less clear is the optimal treatment strategy for patients with limited (1-3) BMs. Surgery and stereotactic radiotherapy are valid options in these patients. The integration of WBRT in this treatment concept is controversial and needs to be decided individually. A possible treatment approach for the management of BMs has recently been published as part of a review by Bertolini et al. [45]. A collaborative registry study for breast cancer patients with central nervous system metastases has been established in Germany, and it is strongly recommended to enroll patients in this study.

\section{Supportive Care}

The use of the granulocyte colony-stimulating factors (G-CSFs), filgrastim and pegfilgrastim, where indicated, is well established. In a comparative effectiveness study, pegfilgrastim prophylaxis was associated with a reduced risk of neutropenia-related or all-cause hospitalization relative to filgrastim prophylaxis [46]. A recent study in high-risk breast cancer demonstrated that $6 \mathrm{mg}$ lipegfilgrastim, a novel glyco-pegylated G-CSF, was as effective as pegfilgrastim in reducing neutropenia in patients with breast cancer receiving myelosuppressive chemotherapy (LoE 1b/B/AGO +) [47]. Concerning prophylaxis of delayed chemotherapy-induced emesis, dexamethasone was not superior to aprepitant but instead had similar efficacy and toxicity in preventing delayed emesis in breast cancer patients treated with anthracycline plus cyclophosphamide chemotherapy and receiving the same antiemetic prophylaxis for acute emesis $(\mathrm{LoE} 1 \mathrm{~b} / \mathrm{A} / \mathrm{AGO}++)$ [48]. Finally, it was acknowledged and confirmed that combined standard oncology care and palliative care should be considered early in the course of illness for patients with metastatic breast cancer and/or high symptom burden [49]. 


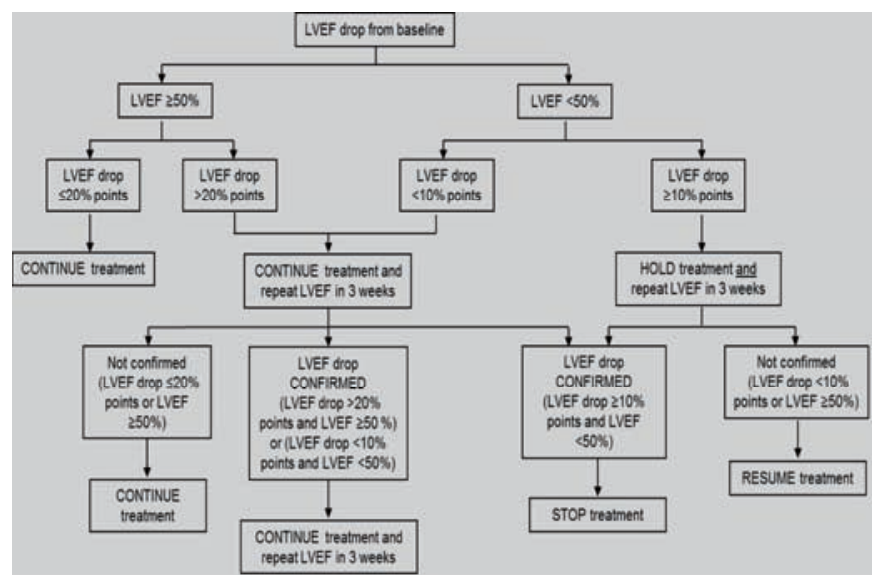

Fig. 4. Algorithm for treatment of trastuzumab/pertuzumab-associated cardiotoxicity.

In the last decade, major concerns were raised regarding the use of erythropoiesis-stimulating agents (ESAs) in the treatment of chemotherapy-induced anemia (CIA). Inconsistent results were reported especially in the metastatic setting and by meta-analyses [50-52]. In contrast, no data were available for the adjuvant treatment of breast cancer patients. In the last year, 3 major trials reporting results of the use of ESAs in the adjuvant setting have been published [53-55]. With the exception of the known elevated risk of thrombotic complications, these studies showed no negative impact of the use of ESAs on disease progression or mortality, indicating that ESAs are safe for the prevention and treatment of CIA in the adjuvant situation. However, these results are contradictory to guidelines in Canada and the USA and to the pharmaceutical manufacturers' product labels which indicate that these agents should not be used when patients receive chemotherapy with curative intent.

\section{Therapy Side Effects}

Acute toxicity and (in most cases) 100-day mortality rates are well documented in the majority of phase III trials. Toxicities are graded according to World Health Organization or National Cancer Institute standards. Various cytotoxic anticancer drugs have their class-specific toxicity profiles. Anthracycline-based standard chemotherapy regimens in the adjuvant setting demonstrate rela- tively low acute toxicity, and treatment-related mortality rates are below $1 \%$. However, with respect to long-term side effects, cardiotoxicity is clinically relevant. In addition, the impact of the biological age on adjuvant decision-making has to be considered, e.g. by measuring the biological age using the comprehensive geriatric assessment (CGA) [56]. The risk of cardiotoxicity associated with trastuzumab has been reported to be $4 \%$ in monotherapy and $27 \%$ when administered in combination with anthracyclines and cyclophosphamide; however, life-threatening or severe adverse events are rare [57].

With respect to cardiac toxicity of treatment combinations, see the algorithm in figure 4 (based on the Affinity/SAKK 2210 study protocol, SAKK = Schweizerische Arbeitsgemeinschaft für Klinische Krebsforschung, Swiss Oncology Research Network). The combination of pertuzumab and trastuzumab plus docetaxel did not increase the incidence of cardiac adverse events (CLEOPATRA study) [58].

The NeoALTTO study introduced lapatinib and trastuzumab in the neoadjuvant setting together with paclitaxel without major cardiac dysfunction, but a high incidence of diarrhea due to lapatinib was noted [59]. Several neuropsychological studies suggest an association between chemotherapy and long-lasting cognitive deficits, possibly related to therapy-induced structural and functional alterations in the brain [60].

\section{Supplementary Material}

Appendix: Members of the 'AGO Breast Committee' in alphabetical order. To access the supplemental material please refer to www.karger. $\mathrm{com} /$ ?DOI $=431248$.

\section{Disclosure Statement}

C.L. has received consultant and speaker honoraria from Amgen, Celgene, Genomic Health, GSK, Novartis, Pierre-Fabre, Roche, and TEVA. M.T. has received consultant and speaker honoraria from AstraZeneca, Amgen, Celgene, Genomic Health, GSK, Novartis, Pierre-Fabre, Serag-Wiessner, Roche, and Sysmex Europe. V.H. has received consultant and speaker honoraria from Novartis and Roche. F.S. has received consultant and speaker honoraria from AstraZeneca, Amgen, Celgene, Novartis, Roche Pharma, and medtronic. The authors have no relevant affiliation or financial involvement with any organization or entity with financial interest in or financial conflict with the subject matter or materials discussed in the manuscript apart from the disclosed.

\section{References}

1 Centre for Evidence Based Medicine: Oxford Level of Evidence, LoE. www.cebm.net.

2 www.ago-online.de/de/fuer-mediziner/leitlinienempfehlungen/

3 Hortobagyi GN, Lipton A, Chew HK, et al.: Efficacy and safety of continued zoledronic acid every 4 weeks versus every 12 weeks in women with bone metastases from breast cancer: results of the OPTIMIZE-2 trial. J Clin Oncol 2014;32(suppl):5s(abstr LBA9500).
4 Hurwitz MD, Ghanouni P, Kanaev SV, et al.: Magnetic resonance-guided focused ultrasound for patients with painful bone metastases: phase III trial results. J Natl Cancer Inst 2014;106:pii:dju082.

5 Schilcher J, Koeppen V, Aspenberg P, Michaelsson K: Risk of atypical femoral fracture during and after bisphosphonate use. Acta Orthop 2015;86:100-107.

6 Cardoso F, Costa A, Norton L, et al.: ESO-ESMO 2nd international consensus guidelines for advanced breast cancer (ABC2). Breast 2014;23:489-502.
7 Chan DS, Vieira AR, Aune D, et al.: Body mass index and survival in women with breast cancer - systematic literature review and meta-analysis of 82 follow-up studies. Ann Oncol 2014;25:1901-1914.

8 Drukker CA, Elias SG, Nijenhuis MV, et al.: Gene expression profiling to predict the risk of locoregional recurrence in breast cancer: a pooled analysis. Breast Cancer Res Treat 2014;148:599-613. Erratum in: Breast Cancer Res Treat 2015;149:567. 
9 Fitzal F, Filipits M, Fesl C, et al.: Predicting local recurrence using PAM50 in postmenopausal endocrine responsive breast cancer patients. J Clin Oncol 2014;32 (suppl):5s(abstr 1008).

10 Uth CC, Christensen MH, Oldenbourg MH, et al.: Sentinel lymph node dissection in locally recurrent breast cancer. Ann Surg Oncol 2015;Epub ahead of print

11 Aebi S, Gelber S, Anderson SJ, et al.; CALOR investigators: Chemotherapy for isolated locoregional recurrence of breast cancer (CALOR): a randomised trial. Lancet Oncol 2014;15:156-163.

12 Aurilio G, Disalvatore D, Pruneri G, et al.: A metaanalysis of oestrogen receptor, progesterone receptor and human epidermal growth factor receptor 2 discordance between primary breast cancer and metastases. Eur J Cancer 2014;50:277-289.

13 Jonat W, Kaufmann M, Blamey RW, et al.: A randomised study to compare the effect of the luteinising hormone releasing hormone (LHRH) analogue goserelin with or without tamoxifen in pre- and peri-menopausal patients with advanced breast cancer. Eur J Cancer 1995;31A:137-142.

14 Gibson L, Lawrence D, Dawson C, Bliss J: Aromatase inhibitors for treatment of advanced breast cancer in postmenopausal women. Cochrane Database Syst Rev 2009;(4):CD003370.

15 Di Leo A, Jerusalem G, Petruzelka L, et al.: Results of the CONFIRM phase III trial comparing fulvestrant $250 \mathrm{mg}$ with fulvestrant $500 \mathrm{mg}$ in postmenopausal women with estrogen receptor-positive advanced breast cancer. J Clin Oncol 2010;28:4594-4600.

16 Baselga J, Campone M, Piccart M, et al.: Everolimus in postmenopausal hormone-receptor-positive advanced breast cancer. N Engl J Med 2012;366:520-529.

17 Finn RS, Crown JP, Lang I, et al.: The cyclin-dependent kinase 4/6 inhibitor palbociclib in combination with letrozole versus letrozole alone as first-line treatment of oestrogen receptor-positive, HER2-negative, advanced breast cancer (PALOMA-1/TRIO-18): a randomised phase 2 study. Lancet Oncol 2015;16:25-35.

18 Martín M, Loibl S, von Minckwitz G, et al.: Phase III trial evaluating the addition of bevacizumab to endocrine therapy as first-line treatment for advanced breast cancer: the letrozole/fulvestrant and avastin (LEA) study. J Clin Oncol 2015;33:1045-1052.

19 Kaufman B, Mackey JR, Clemens MR, et al.: Trastuzumab plus anastrozole versus anastrozole alone for the treatment of postmenopausal women with human epidermal growth factor receptor 2-positive, hormone receptor-positive metastatic breast cancer: results from the randomized phase III TAnDEM study. J Clin Oncol 2009;27:5529-5537.

20 Cardoso F, Costa A, Norton L, et al.: ESO-ESMO 2nd international consensus guidelines for advanced breast cancer (ABC2). Ann Oncol 2014;25:1871-1888.

21 Dear RF, McGeechan K, Jenkins MC, et al.: Combination versus sequential single agent chemotherapy for metastatic breast cancer. Cochrane Database Syst Rev 2013;12:Cd008792.

22 Gligorov J, Doval D, Bines J, et al.: Maintenance capecitabine and bevacizumab versus bevacizumab alone after initial first-line bevacizumab and docetaxel for patients with HER2-negative metastatic breast cancer (IMELDA): a randomised, open-label, phase 3 trial. Lancet Oncol 2014;15:1351-1360.

23 Bidard FC, Peeters DJ, Fehm T, et al.: Clinical validity of circulating tumour cells in patients with metastatic breast cancer: a pooled analysis of individual patient data. Lancet Oncol 2014;15:406-414.

24 Bidard FC, Fehm T, Ignatiadis M, et al.: Clinical application of circulating tumor cells in breast cancer: overview of the current interventional trials. Cancer Metastasis Rev 2013;32:179-188.
25 Wang X, Huang C, Li M, et al.: The efficacy of bevacizumab plus paclitaxel as first-line treatment for HER2negative metastatic breast cancer: a meta-analysis of randomized controlled trials. Tumour Biol 2014;35: 4841-4848.

26 Von Minckwitz G, Puglisi F, Cortes J, et al.: Bevacizumab plus chemotherapy versus chemotherapy alone as second-line treatment for patients with HER2-negative locally recurrent or metastatic breast cancer after first-line treatment with bevacizumab plus chemotherapy (TANIA): an open-label, randomised phase 3 trial. Lancet Oncol 2014;15:1269-1278.

27 Tutt A, Ellis P, Kilburn L, et al.: TNT: A randomized phase III trial of carboplatin (C) compared with docetaxel (D) for patients with metastatic or recurrent locally advanced triple negative or BRCA1/2 breast cancer (CRUK/07/012). San Antonio Breast Cancer Symposium 2014; abstr S3-01.

28 Swain SM, Baselga J, Kim SB, et al.: Pertuzumab, trastuzumab, and docetaxel in HER2-positive metastatic breast cancer. N Engl J Med 2015;372:724-734.

29 Verma S, Miles D, Gianni L, et al.: Trastuzumab emtansine for HER2-positive advanced breast cancer. N Engl J Med 2012;367:1783-1791.

30 Krop IE, Kim S-B, González-Martín A, et al.: Trastuzumab emtansine versus treatment of physician's choice for pretreated HER2-positive advanced breast cancer (TH3RESA): a randomised, open-label, phase 3 trial. Lancet Oncol 2014;15:689-699.

31 Khan SA, Stewart AK, Morrow M: Does aggressive local therapy improve survival in metastatic breast cancer? Surgery 2002;132:620-626.

32 Badwe R, Parmar V, Hawaldar R, et al.: Surgical removal of primary tumor and axillary lymph nodes in women with metastatic breast cancer at first presentation: a randomized controlled trial. San Antonio Breast Cancer Symposium 2013;abstr S2-02. www.sabcs. org/ pastsymposia/index.asp.

33 Rapiti E, Verkooijen HM, Vlastos G, et al.: Complete excision of primary breast tumor improves survival of patients with metastatic breast cancer at diagnosis. J Clin Oncol 2006;24:2743-2749.

34 Babiera GV, Rao R, Feng L, et al.: Effect of primary tumor extirpation in breast cancer patients who present with stage IV disease and an intact primary tumor. Ann Surg Oncol 2006;13:776-782.

35 Howlader M, Heaton N, Rela M: Resection of liver metastases from breast cancer: towards a management guideline. Int J Surg 2011;9:285-291.

36 Abbott DE, Brouquet A, Mittendorf EA, et al.: Resection of liver metastases from breast cancer: estrogen receptor status and response to chemotherapy before metastasectomy define outcome. Surgery 2012;151:710-716.

37 Hoffmann RT, Jakobs TF, Kubisch CH, et al.: Radiofrequency ablation after selective internal radiation therapy with yttrium 90 microspheres in metastatic liver disease - is it feasible? Eur J Radiol 2010;74:199-205.

38 Vogl TJ, Farshid P, Naguib NN, Zangos S: Thermal ablation therapies in patients with breast cancer liver metastases: a review. Eur Radiol 2013;23:797-804.

39 Rashid OM, Takabe K: The evolution of the role of surgery in the management of breast cancer lung metastasis. J Thorac Dis 2012;4:420-424.

40 Demmy TL: Optimal management of malignant pleural effusions (results of CALBG 30102). J Natl Compr Canc Netw 2012;10:975-982.

41 Sebastian M, Kuemmel A, Schmidt M, Schmittel A: Catumaxomab: a bispecific trifunctional antibody. Drugs Today (Barc) 2009;45:589-597.

42 Cozzi S, Montanara S, Luraschi A, et al.: Management of neoplastic pericardial effusions. Tumori 2010;96: 926-929.
43 Kopp HG, Krauss K, Fehm T, et al.: Symptomatic bone marrow involvement in breast cancer - clinical presentation, treatment, and prognosis: a single institution review of 22 cases. Anticancer Res 2011;31:4025-4030.

44 Sperduto PW, Kased N, Roberge D, et al.: Summary report on the graded prognostic assessment: an accurate and facile diagnosis-specific tool to estimate survival for patients with brain metastases. J Clin Oncol 2012;30:419-425.

45 Bertolini F, Spallanzani A, Fontana A, et al.: Brain metastases: an overview. CNS Oncol 2015;4:37-46.

46 Naeim A, Henk HJ, Becker L, et al.: Pegfilgrastim prophylaxis is associated with a lower risk of hospitalization of cancer patients than filgrastim prophylaxis: a retrospective United States claims analysis of granulocyte colonystimulating factors (G-CSF). BMC Cancer 2013;13:11.

47 Bondarenko I, Gladkov OA, Elaesser R, et al.: Efficacy and safety of lipegfilgrastim versus pegfilgrastim: a randomized, multicenter, active-control phase 3 trial in patients with breast cancer receiving doxorubicin/ docetaxel chemo- therapy. BMC Cancer 2013;13:386.

48 Roila F, Ruggeri B, Ballatori E, et al.: Aprepitant versus dexamethasone for preventing chemotherapy-induced delayed emesis in patients with breast cancer: a randomized double-blind study. J Clin Oncol 2014;32: 101-106.

49 Smith TJ, Temin S, Alesi ER, et al.: American Society of Clinical Oncology provisional clinical opinion: the integration of palliative care into standard oncology care. J Clin Oncol 2012;30:880-887.

50 Leyland-Jones B, Semiglazov V, Pawlicki M, et al.: Maintaining normal hemoglobin levels with epoetin alfa in mainly nonanemic patients with metastatic breast cancer receiving first-line chemotherapy: a survival study. J Clin Oncol 2005;23:5960-5972.

51 Tonelli M, Hemmelgarn B, Reimann T, et al.: Benefits and harms of erythropoiesis-stimulating agents for anemia related to cancer. A meta-analysis. CMAJ 2009; 180:E62-E71.

52 Glaspy J, Crawford J, Vansteenkiste J, et al.: Erythropoiesis-stimulating agents in oncology: a study-level meta-analysis of survival and other safety outcomes. $\mathrm{Br}$ J Cancer 2010;102:301-315.

53 Moebus V, Jackisch C, Schneeweiss A, et al.: Adding epoetin alfa to intense dose-dense adjuvant chemotherapy for breast cancer: randomized clinical trial. J Natl Cancer Inst 2013;105:1018-1026.

54 Swain SM, Tang G, Geyer CE, et al.: Definitive results of a phase III adjuvant trial comparing three chemotherapy regimens in women with operable, node-positive breast cancer: the NSABP B-38 trial. J Clin Oncol 2013;31:3197-3204.

55 Nitz U, Gluz O, Oberhoff C, et al.: Final results from the prospective phase III WSG-ARA trial: impact of adjuvant darbepoetin alfa on event-free survival in early breast cancer. Ann Oncol 2014;25:75-80.

56 Pallis AG, Ring A, Fortpied C, et al.: EORTC workshop on clinical trial methodology in older individuals with a diagnosis of solid tumors. Ann Oncol 2011;22;19221926

57 Keefe DL: Traztuzumab-associated cardiotoxicity. Cancer 2002;95;1592-1600.

58 Swain SM, Ewer MS, Cortés J, et al.: Cardiac tolerability of pertuzumab plus trastuzumab plus docetaxel in patients with HER2- positive metastatic breast cancer in CLEOPATRA. Oncologist 2013;18:257-264.

59 Baselga J, Bradbury I, Eidtmann H, et al.: Lapatinib with trastuzumab for HER2-positive early breast cancer (NeoALTTO). Lancet 2012;379;633-640.

60 Koppelmanns V, Breteler MM, Boogerd W, et al.: Late effects of adjuvant chemotherapy for adult onset nonCNS cancer; cognitive impairment, brain structure and risk of dementia. Crit Rev Oncol Hematol 2013;88:87101. 